International Journal of Network Security \& Its Applications (IJNSA), Vol.5, No.4, July 2013

\title{
A Framework for Security Components Anomalies Severity Evaluation and Classification
}

\author{
Kamel Karoui ${ }^{1}$, Fakher Ben Ftima ${ }^{2}$ and Henda Ben Ghezala ${ }^{3}$ \\ ${ }^{1}$ RIADI, ENSI, University of Manouba, Manouba, Tunisia \\ kamel.karoui@insat.rnu.tn \\ ${ }^{2}$ RIADI, ENSI, University of Manouba, Manouba, Tunisia \\ fakher.benftima@infcom.rnu.tn \\ ${ }^{3}$ RIADI, ENSI, University of Manouba, Manouba, Tunisia \\ Henda.bgecck.rnu.tn
}

\begin{abstract}
Security components such as firewalls, IDS and IPS, are the most widely adopted security devices for network protection. These components are often implemented with several errors (or anomalies) that are sometimes critical. To ensure the security of their networks, administrators should detect these anomalies and correct them. Before correcting the detected anomalies, the administrator should evaluate and classify these latter to determine the best strategy to correct them. In this work, we propose a framework to assess and classify the detected anomalies using a three evaluation criteria: a quantitative evaluation, a semantic evaluation and multi-anomalies evaluation. The proposed process, convenient in an audit process, will be detailed by a case study to demonstrate its usefulness.
\end{abstract}

\section{KEYWORDS}

Anomaly severity evaluation, anomaly severity classification, semantic evaluation, quantitative evaluation, multi-anomalies evaluation.

\section{INTRODUCTION}

Rules in a security component can be misconfigured, which implies many conflicts. A misconfiguration or a conflict between rules means that the security component, may either:

- accept some malicious packets, which consequently create security holes.

-discard some legitimate packets, which consequently disrupt normal traffic.

Both cases could cause irreparable consequences. Unfortunately, it has been observed that most security components are implemented with anomalies. Depending on the nature of the anomaly, it can be critical, less critical or benign. Considering the impact of these anomalies on the network security, such errors cannot be tolerated [2].

There are several researches that have proposed for anomalies detection and correction but rare those who are interested in the anomalies severity and their impact on network security. The evaluation and classification of anomalies severity provide the administrator with:

-many correction scenarios

-a classification of rules to be corrected according to their criticality

-an overview of the security component vulnerabilities

DOI : 10.5121/ijnsa.2013.5405 
International Journal of Network Security \& Its Applications (IJNSA), Vol.5, No.4, July 2013

In this paper, we propose firstly an evaluation metrics of the anomalies severity based on the following criteria:

-a quantitative criterion: to get an idea of the number of rules involved in the anomaly.

-a semantic criterion: to assess the impact of the misconfiguration on the services provided (HTTP,FTP,..)

-a multi-anomalies criterion: to study the impact of the composition of many anomalies together.

By combining the three metrics together, we can measure the impact of each anomaly on the security component rule base. For this, we will classify these anomalies severity importance according to their nature, namely; shadowing anomaly, generalization anomaly, redundancy anomaly and correlation anomaly. This classification will determine rules that cause the security component vulnerability.

The remaining parts of the paper are organized as follows: section 1 introduces related works in security component anomalies detection and correction. Section 2 schematizes the proposed approach model. Sections 3 to 5 detail the proposed process steps and section 6 concludes the paper.

\section{RELATED WORKS}

As presented in the introduction, a lot of research has been proposed for the security components analysis, misconfigurations' detection and correction. Most research focuses on the firewalls network policy; in [10], [7] and [6] the authors propose a model for firewalls properties analysis and anomalies detection. Also, the authors of [5] and [4] suggest another model to detect firewalls misconfigurations in central and distributed architectures. In [1], the authors analyze firewall rules using an expert system whereas the authors of [8] analyze firewalls with relational algebra. In [3], the authors put forward a model for IPsec and VPN verification. However, these security components (homogenous or heterogeneous) may conflict when they are installed together on a network. In this context, [2] propose a solution for firewalls and IDS misconfigurations detection.

In reviewing these few references, we note that there is no works that assessed the severity of anomalies before correcting them. The study of the severity can give the administrator more information about the vulnerability of the component. In addition, the classification step exploits this information by illustrating the impact of these errors on the network security by a set of diagrams. In this work, we will develop these two concepts; we begin by detailing our proposed approach in the following section, we will

\section{THE PROPOSED APPROACH}

In order to evaluate and classify the security component anomalies severity, we propose an approach composed of several steps schematized in figure 1. In the case study, we will apply our approach to firewalls. However, the approach should apply for all security components based on filtering attributes. Below, we will briefly present these steps:

\section{-Step A: Security component anomalies detection}

Usually, most security components' base rule contains some misconfigurations. This step consists in checking the security component' base rule to detect anomalies. For the firewall, we enumerate 
International Journal of Network Security \& Its Applications (IJNSA), Vol.5, No.4, July 2013

four kinds of anomalies, namely; shadowing anomaly, generalization anomaly, redundancy anomaly and correlation anomaly. We will not detail this section because it is not the purpose of the paper. For more details, refer to [9]. In the next step, we will evaluate the severity of the detected anomalies (See step A in figure 1).

\section{- Step B: The security component anomalies' severity importance evaluation}

The detected anomalies in step A will be classified into several sub-sets:

- Shadowing anomalies sub-set: contains rules that are shadowed by other rules in the base rule.

-Generalization anomalies sub-set: contains rules that are generalized by other rules in the base rule.

-Redundancy anomalies sub-set: contains rules that are redundant to other rules in the base rule.

-Correlation anomalies sub-set: contains rules that are correlated to other rules in the base rule.

These sub-sets will allow us to evaluate each anomaly severity importance using a three metrics; a quantitative metric, a semantic metric and multi-anomalies metric (See step B in figure 1).

\section{- Step C: Anomalies severity importance classification}

The defined metrics in step B, will be exploited together in order to classify the anomalies severity. We will classify the severity importance according to the shadowing level degree, the generalization level degree, the redundancy level degree and the correlation level degree. (See step $\mathrm{C}$ in figure 1).

\section{-Step D: Security component anomalies correction}

This step consists in correcting the detected anomalies in step B. The correction strategy depends on the classification results returned in step $\mathrm{C}$. We will not detail this section because it is not the purpose of the paper (see step D in figure 1).

In the process presented in figure 1, the gray colored part (steps A and D) is the part already made in several research works. The blue-colored part (steps B and C) is the part that we propose and detail in the following sections. 


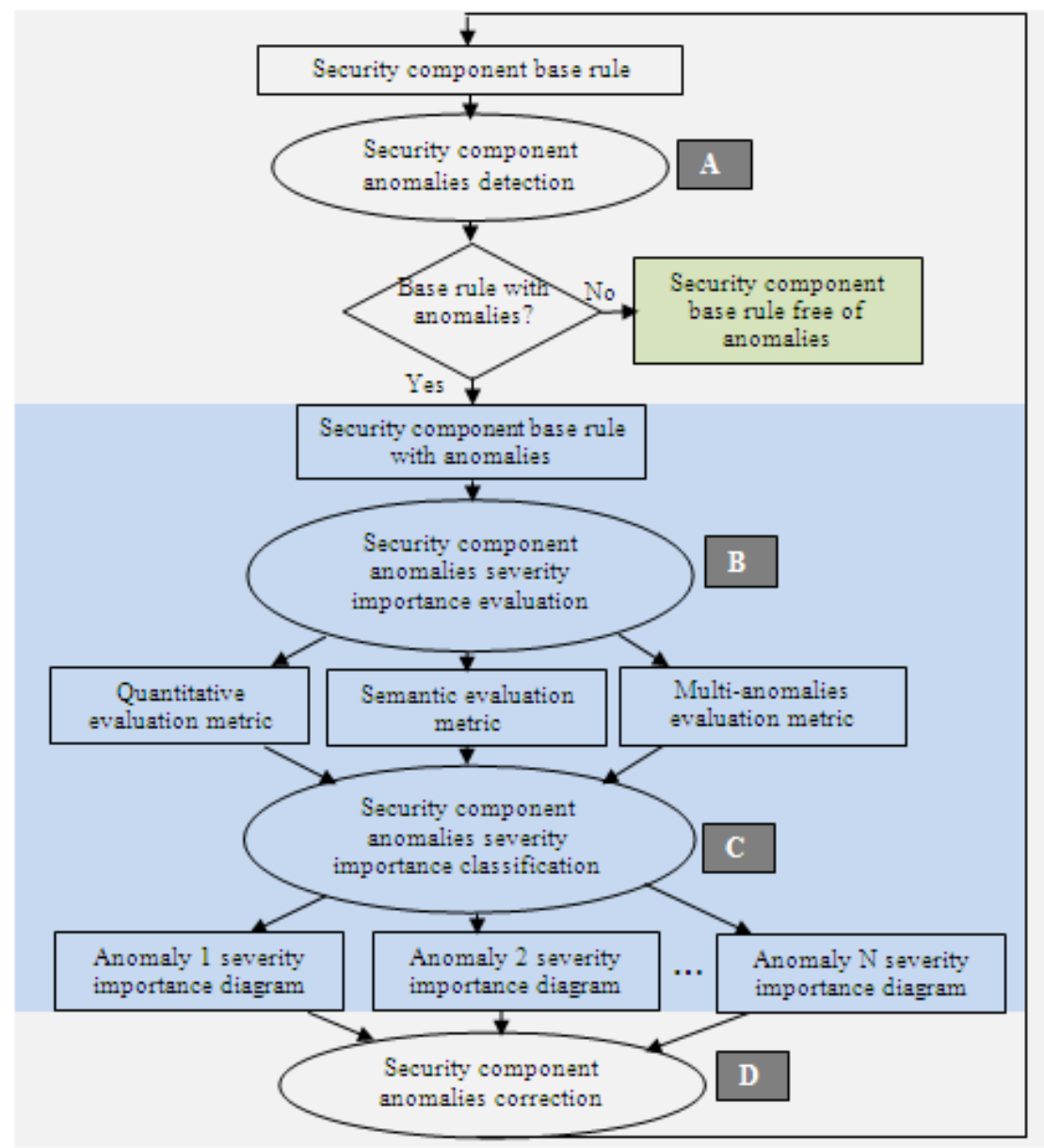

Figure 1. The proposed approach

\section{FORMAL SECURITY COMPONENT'S BASE RULE VERIFICATION (STEP A)}

Generally, security components are specified by a set of formal rules which can be filtering or alerting ones. A rule defines a decision (such as "deny", "alert", "accept", or "pass") that applies over a set of condition attributes (such as, "source address", "destination address", "source port", "destination port", "protocol", "attack class", etc. ). Let's take a security component composed of a set $Q$ of $t$ rules $\left(r_{i} \in Q\right.$ with $1 \leq i \leq t$ ). A rule $r_{i}$ in the set $\mathrm{Q}$ is represented formally as follows:

$$
\mathbf{r}_{\mathrm{i}}: \mathbf{r}_{\mathrm{i}}\left[\mathbf{A}_{1}\right] \mathbf{r}_{\mathrm{i}}\left[\mathbf{A}_{2}\right] \ldots \mathbf{r}_{\mathrm{i}}\left[\mathbf{A}_{\mathbf{n}}\right] \text { where }
$$

$-A_{1}, A_{2} \ldots A_{n-1}$ are the rule $r_{i}$ attributes'. For example, in table 1, the attribute $A_{2}=$ Protocol. $-r_{i}\left[A_{m}\right]$ is the attribute $A_{m}$ value with $(1 \leq m \leq n)$. For example, in table $1, r_{3}\left[A_{2}\right]=T C P$.

- $A_{n}$ is the attribute "Decision". For example, in table $1, A_{7}$ is the decision attribute and $\mathrm{r}_{3}\left[\mathrm{~A}_{7}\right]=$ deny.

For more details about rules formalization, refer to [4]. 
An anomaly in a security component base rule can be the result of the following cases [5]:

-The existence of two or more rules that may match the same packet.

-The existence of a rule that can never be activated. We note that a rule $r_{i}$ is activated if there is an IP packet that was accepted or rejected by applying the rule $r_{i}$.

There are four different anomalies that may exist among rules in a security component base rule, namely:

-Shadowing anomaly: a rule $r_{j}$ is shadowed by a previous rule $r_{i}$ when $r_{i}$ matches all the packets that match $r_{j}$ and the two rules have different decisions, such that the shadowed rule $r_{j}$ will never be activated (see example in section 4.1).

-The generalization anomaly: The generalization anomaly is the reverse of the shadowing anomaly i.e. in a base rule $Q$, a rule $r_{j}$ is a generalization of a preceding rule $r_{i}$ if, on the one hand, the rule $r_{j}$ can match all the packets that match the rule $r_{i}$ and, on the other hand, the two rules have different decisions (see example in section 4.1).

-The redundancy anomaly: In a base rule $Q$, a rule $r_{j}$ is redundant to a rule $r_{i}$ if $r_{j}$ performs the same decision on the same packets as $r_{i}$. In the way, if the redundant rule $r_{j}$ is removed, the safety of the security component will not be affected (see example in section 4.1).

- The correlation anomaly: In a base rule $Q$, the rule $r_{j}$ is correlated to $r_{i}$ if, on the one hand, the first rule $r_{i}$ matches some packets that match the second rule $r_{j}$, and the second rule $r_{j}$ matches some packets that match the first rule $r_{i}$ and, on the other hand, the two rules have different decisions (see example in section 4.1).

For more details about the security component anomalies, refer to [5].

\subsection{Case study}

Let's take a security component $F w$, composed of a base rule $Q$. Each rule $r_{i}$ belonging to $Q$ has the following attributes: "Packet length", "Protocol", "Source address", "Destination address", "Source port", "Destination port" and "Decision".

\begin{tabular}{|c|c|c|c|c|c|c|c|}
\hline Rules & $\begin{array}{l}\text { Packet } \\
\text { length } \\
\left(A_{1}\right)\end{array}$ & $\begin{array}{c}\text { Protocol } \\
\left(A_{2}\right)\end{array}$ & $\begin{array}{c}\text { Source address } \\
\left(A_{s}\right)\end{array}$ & $\begin{array}{l}\text { Src. } \\
\text { Port } \\
\left(\mathrm{A}_{4}\right)\end{array}$ & $\begin{array}{c}\text { Destination address } \\
\left(A_{5}\right)\end{array}$ & $\begin{array}{l}\text { Dest. } \\
\text { Port } \\
\left(A_{1}\right)\end{array}$ & $\begin{array}{c}\text { Decision } \\
\left(A_{7}\right)\end{array}$ \\
\hline $\mathrm{r}_{\mathrm{t}}$ & All & $\mathrm{TCP}$ & {$[140.192 .10 .1-140.192 .10 .100]$} & Any & {$[129.170 .20 .20-129.170 .20 .100]$} & Any & deny \\
\hline $\mathrm{r}_{2}$ & All & $\mathrm{TCP}$ & {$[140.192 .10 .20-140.192 .10 .50]$} & Any & {$[129.170 .20 .20-129.170 .20 .70]$} & 80 & accept \\
\hline$r_{3}$ & AII & $\mathrm{TCP}$ & {$[140.192 .10 .1-140.192 .10 .60]$} & Any & {$[129.170 .20 .20-129.170 .20 .100]$} & Any & deny \\
\hline $\mathrm{r}_{4}$ & 16 & $\mathrm{TCP}$ & {$[140.192 .10 .1-140.192 .10 .100]$} & Any & $129.170 .20 .30-129.170 .20 .100]$ & $80^{\circ}$ & accept \\
\hline $\mathrm{r}_{5}$ & 16 & $\mathrm{TCP}$ & {$[140.192 .10 .20-140.192 .10 .50]$} & Any & $129.170 .20 .30-129.170 .20 .70]$ & 80 & deny \\
\hline$r_{6}$ & 16 & $\mathrm{TCP}$ & {$[140.192 .10 .20-140.192 .10 .50]$} & Any & $129.170 .20 .30-129.170 .20 .70]$ & 80 & accept \\
\hline r7 & All & $\mathrm{TCP}$ & {$[140.192 .10 .20-140.192 .10 .50]$} & 23 & {$[129.170 .20 .30-1129.170 .20 .70]$} & 80 & deny \\
\hline $\mathrm{r}_{\mathrm{s}}$ & 16 & $\mathrm{TCP}$ & {$[140.192 .10 .170-140.192 .10 .200]$} & Any & {$[129.170 .20 .30-129.170 .20 .70]$} & 25 & deny \\
\hline$r_{8}$ & All & $\mathrm{TCP}$ & $140.192 .10 .151-140.192 .10 .200]$ & Any & $129.170 .20 .20-1129.170 .20 .70]$ & 23 & accept \\
\hline$r: 0$ & All & $\mathrm{TCP}$ & {$[140.192 .10 .101-140.192 .10 .150]$} & Any & {$[129.170 .20 .30-129.170 .20 .70]$} & 21 & deny \\
\hline
\end{tabular}

Table 1 . The security component $F w$ base rule

By analyzing the security component $F w$, we note that there some anomalies detailed as follows: -For the shadowing anomaly, we note that $r_{2}$ is shadowed by $r_{1}$. More precisely: 
$\left(r_{2}\left[A_{1}\right]=r_{1}\left[A_{1}\right]\right) \wedge\left(r_{2}\left[A_{2}\right]=r_{1}\left[A_{2}\right]\right) \wedge\left(r_{2}\left[A_{3}\right] \subset r_{1}\left[A_{3}\right]\right) \wedge\left(r_{2}\left[A_{4}\right]=r_{1}\left[A_{4}\right]\right) \wedge$

$\left(r_{2}\left[A_{5}\right] \subset r_{1}\left[A_{5}\right]\right) \wedge\left(r_{2}\left[A_{6}\right] \subset r_{1}\left[A_{6}\right]\right) \wedge\left(r_{2}\left[A_{7}\right] \neq r_{1}\left[A_{7}\right]\right)$

Also, in the same table, we note that $\left(r_{4}\right.$ and $r_{6}$ are shadowed by $\left.r_{1}\right),\left(r_{5}\right.$ and $r_{7}$ are shadowed by $\left.r_{2}\right),\left(r_{6}\right.$ is shadowed by $\left.r_{3}\right),\left(r_{5}\right.$ and $r_{7}$ are shadowed by $\left.r_{4}\right)$ and $\left(r_{6}\right.$ is shadowed by $\left.r_{5}\right)$.

-For the generalization anomaly, we note that $r_{2}$ is generalized by $r_{3}$. More precisely:

$\left(r_{2}\left[A_{1}\right]=r_{3}\left[A_{1}\right]\right) \wedge\left(r_{2}\left[A_{2}\right]=r_{3}\left[A_{2}\right]\right) \wedge\left(r_{2}\left[A_{3}\right] \subset r_{3}\left[A_{3}\right]\right) \wedge\left(r_{2}\left[A_{4}\right]=r_{3}\left[A_{4}\right]\right) \wedge$

$\left(r_{2}\left[A_{5}\right] \subset r_{3}\left[A_{5}\right]\right) \wedge\left(r_{2}\left[A_{6}\right] \subset r_{3}\left[A_{6}\right]\right) \wedge\left(r_{2}\left[A_{7}\right] \neq r_{3}\left[A_{7}\right]\right)$

-For the redundancy anomaly, we note that $r_{3}$ is redundant to $r_{1}$. More precisely:

$\left(r_{3}\left[A_{1}\right]=r_{1}\left[A_{1}\right]\right) \wedge\left(r_{3}\left[A_{2}\right]=r_{1}\left[A_{2}\right]\right) \wedge\left(r_{3}\left[A_{3}\right] \subset r_{1}\left[A_{3}\right]\right) \wedge\left(r_{3}\left[A_{4}\right]=r_{1}\left[A_{4}\right]\right) \wedge$

$\left(r_{3}\left[A_{5}\right]=r_{1}\left[A_{5}\right]\right) \wedge\left(r_{3}\left[A_{6}\right]=r_{1}\left[A_{6}\right]\right) \wedge\left(r_{3}\left[A_{7}\right]=r_{1}\left[A_{7}\right]\right)$

Also, in the same table, we note that $\left(r_{5}\right.$ and $r_{7}$ are redundant to $\left.r_{1}\right),\left(r_{5}\right.$ and $r_{7}$ are redundant to $\left.r_{3}\right)$, $\left(r_{6}\right.$ is redundant to $\left.r_{2}\right)$ and $\left(r_{6}\right.$ is redundant to $\left.r_{4}\right)$.

-For the correlation anomaly, we note that $r_{4}$ is correlated to $r_{3}$. More precisely:

$\left(r_{3}\left[A_{1}\right] \supset r_{4}\left[A_{1}\right]\right) \wedge\left(r_{3}\left[A_{2}\right]=r_{4}\left[A_{2}\right]\right) \wedge\left(r_{3}\left[A_{3}\right] \subset r_{4}\left[A_{3}\right]\right) \wedge\left(r_{3}\left[A_{4}\right]=r_{4}\left[A_{4}\right]\right) \wedge$

$\left(r_{3}\left[A_{5}\right] \supset r_{4}\left[A_{5}\right]\right) \wedge\left(r_{3}\left[A_{6}\right] \supset r_{4}\left[A_{6}\right]\right) \wedge\left(r_{3}\left[A_{7}\right] \neq r_{4}\left[A_{7}\right]\right)$

Also, in the same table, we note that $\left(r_{7}\right.$ is correlated to $\left.r_{6}\right)$ and $\left(r_{7}\right.$ is correlated to $\left.r_{4}\right)$.

In the next section, we will first evaluate the severity importance of these anomalies in order to classify them.

\section{THE SECURITY COMPONENT ANOMALIES SEVERITY IMPORTANCE EVALUATION (STEP B)}

After detecting the security component anomalies (see section 4), we will gather them in four subsets that we will define in the next sub-section. Then, we will evaluate their criticality degree by a set of metrics.

\subsection{Anomalies sub-sets definition}

For each one of the anomalies' categories namely, "Shadowing", "Generalization", "Redundancy" and "Correlation", we associate respectively sub-sets $\mathbf{S}, \mathbf{G}, \mathbf{R}$ and $\mathbf{C}$ which contain rules belonging to that category. Let's take a security component composed of a set $Q$ of $t$ rules $\left(r_{i} \in Q\right.$ with $1 \leq i \leq t$ ). We define these sub-sets as follows:

- The set of shadowing rules:

$S=\left\{r_{i} \in Q\left|\exists r_{j} \in Q\right| \forall 1 \leq m \leq(n-1), r_{j}\left[A_{m}\right] \subseteq r_{i}\left[A_{m}\right] \wedge r_{j}\left[A_{n}\right] \neq r_{i}\left[A_{n}\right]\right.$ with $\left.1 \leq i<j \leq t\right\}$

We note that $|\mathrm{S}|$ is the sub-set $S$ cardinality i.e. the number of shadowed rules.

- The set of generalizing rules:

$G=\left\{r_{\mathrm{j}} \in Q\left|\exists r_{\mathrm{i}} \in Q\right| \forall 1 \leq m \leq(n-1), \mathrm{r}_{\mathrm{i}}\left[\mathrm{A}_{\mathrm{m}}\right] \subseteq \mathrm{r}_{\mathrm{j}}\left[\mathrm{A}_{\mathrm{m}}\right] \wedge r_{\mathrm{j}}\left[\mathrm{A}_{\mathrm{n}}\right] \neq r_{\mathrm{i}}\left[\mathrm{A}_{\mathrm{n}}\right]\right.$ with $\left.1 \leq i<j \leq \mathrm{t}\right\}$ (2)

We note that $|\mathrm{G}|$ is the sub-set $G$ cardinality i.e. the number of generalized rules. 
- The set of redundant rules:

$R=\left\{r_{\mathrm{i}} \in Q\left|\exists \mathrm{r}_{\mathrm{j}} \in Q\right| \forall 1 \leq m \leq(n-1), \mathrm{r}_{\mathrm{j}}\left[\mathrm{A}_{\mathrm{m}}\right] \subseteq \mathrm{r}_{\mathrm{i}}\left[\mathrm{A}_{\mathrm{m}}\right] \wedge \mathrm{r}_{\mathrm{i}}\left[\mathrm{A}_{\mathrm{n}}\right]=\mathrm{r}_{\mathrm{j}}\left[\mathrm{A}_{\mathrm{n}}\right]\right.$ with $\left.1 \leq i<j \leq \mathrm{t}\right\}$

We note that $|\mathrm{R}|$ is the sub-set $R$ cardinality i.e. the number of redundant rules.

- The set of correlated rules:

$C=\left\{\begin{array}{l}r_{i} \in Q\left|\exists r_{j} \in Q\right| \forall 1 \leq m \leq(n-1),\left[\left(r_{i}\left[A_{m}\right] \subset r_{j}\left[A_{m}\right]\right) \vee\left(r_{i}\left[A_{m}\right] \supset r_{j}\left[A_{m}\right]\right) \vee\left(r_{i}\left[A_{m}\right]=r_{j}\left[A_{m}\right]\right)\right] \wedge \\ r_{i}\left[A_{n}\right] \neq r_{j}\left[A_{n}\right] \text { with } 1 \leq i<j \leq t\end{array}\right\}$

We note that $|C|$ is the sub-set $C$ cardinality i.e. the number of correlated rules.

Now, we will define new sub-sets allowing us to evaluate the anomalies' impact of each rule belonging to the defined sub-sets ( $, \mathrm{G}, \mathrm{R}$ and $\mathrm{C})$ on the other rules, as follows:

- For each element $r_{i}$ belonging to $S$, we define the set of shadowed rules:

$\mathrm{S}\left(\mathrm{r}_{\mathrm{i}}\right)=\left\{r_{\mathrm{j}} \in Q \mid \forall 1 \leq m \leq(n-1), \mathrm{r}_{\mathrm{j}}\left[\mathrm{A}_{\mathrm{m}}\right] \subseteq \mathrm{r}_{\mathrm{i}}\left[\mathrm{A}_{\mathrm{m}}\right] \wedge r_{\mathrm{j}}\left[\mathrm{A}_{\mathrm{n}}\right] \neq \mathrm{r}_{\mathrm{i}}\left[\mathrm{A}_{\mathrm{n}}\right]\right.$ with $\left.1 \leq i<j \leq \mathrm{t}\right\}(5)$

- For each element $r_{i}$ belonging to $G$, we define the set of generalized rules:

$\mathrm{G}\left(\mathrm{r}_{\mathrm{i}}\right)=\left\{r_{\mathrm{j}} \in Q \mid \forall 1 \leq m \leq(n-1), \mathrm{r}_{\mathrm{i}}\left[\mathrm{A}_{\mathrm{m}}\right] \subseteq \mathrm{r}_{\mathrm{j}}\left[\mathrm{A}_{\mathrm{m}}\right] \wedge r_{\mathrm{j}}\left[\mathrm{A}_{\mathrm{n}}\right] \neq r_{\mathrm{i}}\left[\mathrm{A}_{\mathrm{n}}\right]\right.$ with $\left.1 \leq i<j \leq \mathrm{t}\right\}$ (6)

- For each element $r_{i}$ belonging to $R$, we define the set of redundant rules:

$\mathrm{R}\left(\mathrm{r}_{\mathrm{i}}\right)=\left\{r_{\mathrm{j}} \in Q \mid \forall 1 \leq m \leq(n-1), \mathrm{r}_{\mathrm{j}}\left[\mathrm{A}_{\mathrm{m}}\right] \subseteq \mathrm{r}_{\mathrm{i}}\left[\mathrm{A}_{\mathrm{m}}\right] \wedge \mathrm{r}_{\mathrm{i}}\left[\mathrm{A}_{\mathrm{n}}\right]=r_{\mathrm{j}}\left[\mathrm{A}_{\mathrm{n}}\right]\right.$ with $\left.1 \leq i<j \leq \mathrm{t}\right\}$ (7)

- For each element $r_{i}$ belonging to $C$, we define the set of correlated rules:

$\mathrm{C}\left(\mathrm{r}_{\mathrm{i}}\right)=\left\{\begin{array}{l}r_{\mathrm{j}} \in Q \mid \forall 1 \leq m \leq(n-1),\left[\left(\mathrm{r}_{\mathrm{i}}\left[\mathrm{A}_{\mathrm{m}}\right] \subset \mathrm{r}_{\mathrm{j}}\left[\mathrm{A}_{\mathrm{m}}\right]\right) \vee\left(\mathrm{r}_{\mathrm{i}}\left[\mathrm{A}_{\mathrm{m}}\right] \supset \mathrm{r}_{\mathrm{j}}\left[\mathrm{A}_{\mathrm{m}}\right]\right) \vee\left(\mathrm{r}_{\mathrm{i}}\left[\mathrm{A}_{\mathrm{m}}\right]=\mathrm{r}_{\mathrm{j}}\left[\mathrm{A}_{\mathrm{m}}\right]\right)\right] \wedge \\ r_{\mathrm{i}}\left[\mathrm{A}_{\mathrm{n}}\right] \neq r_{\mathrm{j}}\left[\mathrm{A}_{\mathrm{n}}\right] \text { with } 1 \leq i<j \leq \mathrm{t}\end{array}\right\}$

\subsubsection{Case study}

Applying the four sub-sets (1),(2),(3) and (4) defined above, on the example in Table 1, we obtain the following results:

- $\mathrm{S}=\left\{\mathrm{r}_{1}, \mathrm{r}_{2}, \mathrm{r}_{3}, \mathrm{r}_{4}, \mathrm{r}_{5}\right\}$. We can verify that rules in the sub-set $S$ are rules that are shadowing other rules in the set $\mathrm{Q}$.

- $\mathrm{G}=\left\{\mathrm{r}_{3}\right\}$. We can verify that rules in the sub-set $G$ are rules that are generalizing other rules in the set $Q$.

- $\mathrm{R}=\left\{\mathrm{r}_{1}, \mathrm{r}_{2}, \mathrm{r}_{3}, \mathrm{r}_{4}\right\}$. We can verify that rules in the sub-set $R$ are rules that are redundant to other rules in the set $Q$.

- $\mathrm{C}=\left\{\mathrm{r}_{3}, \mathrm{r}_{4}, \mathrm{r}_{6}\right\}$. We can verify that rules in the sub-set $C$ are rules that are correlating other rules in the set $Q$.

Taking into account the defined sub-sets S, G, R and C, we will apply (5),(6),(7) and (8) on the example in Table 1. We obtain the following results:

- Rules subjected to the shadowing anomaly (see Table 1) as are classified follows: $S\left(r_{1}\right)=\left\{r_{2}, r_{4}, r_{6}\right\}$ We can verify that rules in the sub-set $S\left(r_{1}\right)$ are rules that are shadowed by $r_{1}$ in the set $Q$. 
International Journal of Network Security \& Its Applications (IJNSA), Vol.5, No.4, July 2013

$S\left(r_{2}\right)=\left\{r_{5}, r_{7}\right\}$ We can verify that rules in the sub-set $S\left(r_{2}\right)$ are rules that are shadowed by $r_{2}$ in the set $Q$.

$S\left(r_{3}\right)=\left\{r_{6}\right\}$ We can verify that rules in the sub-set $S\left(r_{3}\right)$ are rules that are shadowed by $r_{3}$ in the set $Q$.

$S\left(r_{4}\right)=\left\{r_{5}, r_{7}\right\}$ We can verify that rules in the sub-set $S\left(r_{4}\right)$ are rules that are shadowed by $r_{4}$ in the set $Q$.

$S\left(r_{5}\right)=\left\{r_{6}\right\}$ We can verify that rules in the sub-set $S\left(r_{5}\right)$ are rules that are shadowed by $r_{5}$ in the set $Q$.

-Rules subjected to the generalization anomaly (see Table 1) are classified as follows:

$G\left(r_{3}\right)=\left\{r_{2}\right\}$ We can verify that rules in the sub-set $G\left(r_{3}\right)$ are rules that are generalized by $r_{3}$ in the set $Q$.

-Rules subjected to the redundant anomaly (see Table 1) are classified as follows:

$R\left(r_{1}\right)=\left\{r_{3}, r_{5}, r_{7}\right\}$ We can verify that rules in the sub-set $R\left(r_{1}\right)$ are rules that are redundant to $r_{1}$ in the set $Q$.

$R\left(r_{3}\right)=\left\{r_{5}, r_{7}\right\}$ We can verify that rules in the sub-set $R\left(r_{3}\right)$ are rules that are redundant to $r_{3}$ in the set $Q$.

$R\left(r_{2}\right)=\left\{r_{6}\right\}$ We can verify that rules in the sub-set $R\left(r_{2}\right)$ are rules that are redundant to $r_{2}$ in the set $Q$.

$R\left(r_{4}\right)=\left\{r_{6}\right\}$ We can verify that rules in the sub-set $R\left(r_{4}\right)$ are rules that are redundant to $r_{4}$ in the set $Q$.

-Rules subjected to the correlation anomaly (see Table 1) are classified as follows:

$C\left(r_{3}\right)=\left\{r_{4}\right\}$ We can verify that rules in the sub-set $C\left(r_{3}\right)$ are rules that are correlated to $r_{3}$ in the set $Q$.

$C\left(r_{6}\right)=\left\{r_{7}\right\}$ We can verify that rules in the sub-set $C\left(r_{6}\right)$ are rules that are correlated to $r_{6}$ in the set $Q$.

$C\left(r_{4}\right)=\left\{r_{7}\right\}$ We can verify that rules in the sub-set $C\left(r_{4}\right)$ are rules that are correlated to $r_{4}$ in the set $Q$.

\subsection{Anomalies severity importance evaluation}

In this section, we will evaluate the anomalies severity regarding three criteria; namely quantitative, semantic and multi-anomalies.

\subsubsection{Quantitative importance evaluation}

The quantitative evaluation is a metric based on the number of rules involved in the anomaly. The cardinality of the four sub-sets ( S, G, R and C) can give us some indications of these anomalies importance. 
For that, we associate a quantitative coefficients $\mathrm{M}_{\mathrm{S}}, \mathrm{M}_{\mathrm{G}}, \mathrm{M}_{\mathrm{R}}$ and $\mathrm{M}_{\mathrm{C}}$ to each element $r_{i}$ belonging respectively to the sub-set $S, G, R$ and $C$. These coefficients express the quantitative importance of each type of error. They are defined as follows:

$$
M_{s}\left(r_{i}\right)=\frac{\left|S\left(r_{i}\right)\right|}{t-1}(9) ; M_{G}\left(r_{i}\right)=\frac{\left|G\left(r_{i}\right)\right|}{t-1}(10) ; M_{R}\left(r_{i}\right)=\frac{\left|R\left(r_{i}\right)\right|}{t-1}(11) \text { and } M_{c}\left(r_{i}\right)=\frac{\left|C\left(r_{i}\right)\right|}{t-1}(12) \text { where } t \text { is the }
$$

number of rules in the set $Q$.

\subsubsection{Case study}

In our case study, the shadowing anomaly coefficient (9) is defined as follows:

$$
\begin{aligned}
& M_{S}\left(r_{1}\right)=\frac{\left|S\left(r_{1}\right)\right|}{t-1}=\frac{3}{9}=0,333 ; \quad M_{s}\left(r_{2}\right)=\frac{\left|S\left(r_{2}\right)\right|}{t-1}=\frac{2}{9}=0,222 ; \quad M_{s}\left(r_{3}\right)=\frac{\left|S\left(r_{3}\right)\right|}{t-1}=\frac{1}{9}=0,111 ; \\
& M_{S}\left(r_{4}\right)=\frac{\left|S\left(r_{4}\right)\right|}{t-1}=\frac{2}{9}=0,222 ; \quad M_{s}\left(r_{5}\right)=\frac{\left|S\left(r_{5}\right)\right|}{t-1}=\frac{1}{9}=0,111
\end{aligned}
$$

The generalization anomaly coefficient (10) is defined as follows:

$$
M_{G}\left(r_{3}\right)=\frac{\left|G\left(r_{3}\right)\right|}{t-1}=\frac{1}{9}=0,111
$$

The redundancy anomaly coefficient (11) is defined as follows:

$$
\begin{aligned}
& M_{R}\left(r_{1}\right)=\frac{\left|R\left(r_{1}\right)\right|}{t-1}=\frac{3}{9}=0,333 ; \quad M_{R}\left(r_{2}\right)=\frac{\left|R\left(r_{2}\right)\right|}{t-1}=\frac{1}{9}=0,111 ; \quad M_{R}\left(r_{3}\right)=\frac{\left|R\left(r_{3}\right)\right|}{t-1}=\frac{2}{9}=0,222 ; \\
& M_{R}\left(r_{4}\right)=\frac{\left|R\left(r_{1}\right)\right|}{t-1}=\frac{1}{9}=0,111
\end{aligned}
$$

Finally, the correlation anomaly coefficient (12) is defined as follows:

$$
M_{c}\left(r_{3}\right)=\frac{\left|C\left(r_{3}\right)\right|}{t-1}=\frac{1}{9}=0,111 ; M_{c}\left(r_{4}\right)=\frac{\left|C\left(r_{4}\right)\right|}{t-1}=\frac{1}{9}=0,111 ; M_{c}\left(r_{6}\right)=\frac{\left|C\left(r_{6}\right)\right|}{t-1}=\frac{1}{9}=0,111
$$

Taking into account the quantitative evaluation criterion, the shadowing anomaly coefficient $\mathrm{M}_{\mathrm{S}}\left(\mathrm{r}_{1}\right)$ is greater than the other shadowing anomaly coefficients seeing that $r_{I}$ shadows more rules. Thus, the shadowing error is more important and will have higher priority in the correction process. Generally, correcting the most important shadowing rule decreases the number of shadowed rules.

As long as, the redundancy anomaly coefficient $\mathrm{M}_{\mathrm{R}}\left(\mathrm{r}_{1}\right)$ is greater than the other redundancy anomaly coefficients seeing that $r_{l}$ is redundant to more rules. Therefore, the redundancy error is also important and will have higher priority in the correction process.

As defined below, the quantitative coefficient $\mathrm{M}$ is based on the number of rules of each sub-set. Although it gives us a first indicator of the anomalies severity importance, nevertheless, some reserves can be expressed: 
- The indicators $M_{R}\left(r_{i}\right)$ or $M_{G}\left(r_{i}\right)$ can be very bad (approximate to 1 ) but not really critical seeing that this rule can be rarely activated.

- In the same way, the indicators $M_{S}\left(r_{i}\right)$ or $M_{C}\left(r_{i}\right)$ can be very good (approximate to 0 ) but probably points out a serious problem if this rule is often activated.

To remedy to the previous reserves, we propose a complementary metric called semantic evaluation. This metric takes into account the semantic of the services involved in the anomaly and gives us an overview of the rule vulnerability degree.

\subsubsection{Semantic importance evaluation}

To propose such a metric, the administrator will order rules regarding one or more filtering attributes (except the attribute "Decision"). As an example, for an e-commerce website, the administrator will give importance to the port 8080 . For an FTP server, it will give importance to the port 23 and 25.

Let choose for example the attribute "destination port" which is, generally, the most important service among the others attributes. In this case, the administrator must classify services offered by the network according to the importance of "destination port" number.

From this classification, we bind each rule to the service to which it is referred and associate an indicator relating to the importance of that service. Let's suppose that we have $\mathrm{z}$ services in the network and a rule $r_{i}$ using to a service classified $k^{\text {th }}$ by the administrator. We associate to $r_{i}$ the value:

$$
v\left(r_{i}\right)=z-k+1 \text { with } 1 \leq k \leq t
$$

If the attribute value is "ANY", this means that this attribute can take any services value provided by the network. For this, it is assigned by the value of the best classified service plus one.

Based on $v\left(r_{i}\right)$, we define semantic evaluation coefficients $\mathrm{M}_{\mathrm{S}}{ }^{\prime}, \mathrm{M}_{\mathrm{G}}{ }^{\prime}, \mathrm{M}_{\mathrm{R}}{ }^{\prime}$ and $\mathrm{M}_{\mathrm{C}}{ }^{\prime}$ for respectively the defined sub-sets $\mathrm{S}, \mathrm{G}, \mathrm{R}$ and $\mathrm{C}$ as follows :

$$
\begin{array}{ll}
M_{s}^{\prime}\left(r_{i}\right)=\frac{v\left(r_{i}\right)}{\operatorname{Max}\left(v\left(r_{i}\right)\right)+1} \quad(13) \text { where } r_{i} \text { belongs to the sub-set } S \\
M_{G}^{\prime}{ }_{G}\left(r_{i}\right)=\frac{v\left(r_{i}\right)}{\operatorname{Max}\left(v\left(r_{i}\right)\right)+1} \quad(14) \text { where } r_{i} \text { belongs to the sub-set } G \\
M_{R}^{\prime}\left(r_{i}\right)=\frac{v\left(r_{i}\right)}{\operatorname{Max}\left(v\left(r_{i}\right)\right)+1} \quad \text { (15) where } r_{i} \text { belongs to the sub-set } R \\
M_{C}^{\prime}{ }_{C}\left(r_{i}\right)=\frac{v\left(r_{i}\right)}{\operatorname{Max}\left(v\left(r_{i}\right)\right)+1} \quad \text { (16) where } r_{i} \text { belongs to the sub-set C }
\end{array}
$$

\subsubsection{Case study}

Based on the defined metrics (13), (14), (15) and (16), we suppose that the administrator has classified the services offered by the attribute "Destination Port ". We notice that, in our case study, the filtering rules use four destination ports (see Table 1) that are; the HTTP port (80), the FTP port (21), the TELNET port (23) and the SMTP port (25) classified by importance as follows: 
International Journal of Network Security \& Its Applications (IJNSA), Vol.5, No.4, July 2013

1. HTTP (this service will have the value $4-1+1=4$ )

2. SMTP (this service will have the value $4-2+1=3$ )

3. TELNET (this service will have the value $4-3+1=2$ )

4. FTP (this service will have the value $4-4+1=1$ )

The service ANY, will have the value of the best classified service plus one. In our case, it will have the value 5 .

In our case, the associated values to each rule representing a given service are the following: $v\left(r_{2}\right)=v\left(r_{4}\right)=v\left(r_{5}\right)=v\left(r_{6}\right)=v\left(r_{7}\right)=4$ since $r_{2}, r_{4}, r_{5}, r_{6}$ and $r_{7}$ are related to the HTTP port.

$v\left(r_{9}\right)=2$ since $r_{9}$ is related to the TELNET port.

$v\left(r_{8}\right)=3$ since $r_{8}$ is related to the SMTP port.

$v\left(r_{10}\right)=1$ since $r_{10}$ is related to the FTP port.

$v\left(r_{1}\right)=v\left(r_{3}\right)=5 r_{1}$ and $r_{3}$ are related to any port.

The four metrics sub-sets $\mathrm{M}_{\mathrm{S}}{ }^{\prime}, \mathrm{M}_{\mathrm{G}}{ }^{\prime}, \mathrm{M}_{\mathrm{R}}$ ' and $\mathrm{M}_{\mathrm{C}}$ ' are calculated as follows:

$$
\begin{aligned}
& M_{S}^{\prime}\left(r_{1}\right)=M_{R}^{\prime}\left(r_{1}\right)=\frac{5}{6}=0,83 ; M_{S}^{\prime}\left(r_{2}\right)=M_{R}^{\prime}\left(r_{2}\right)=\frac{4}{6}=0,66 ; \\
& M_{S}{ }^{\prime}\left(r_{3}\right)=M_{G}{ }^{\prime}\left(r_{3}\right)=M_{R}{ }^{\prime}\left(r_{3}\right)=M_{C}{ }^{\prime}\left(r_{3}\right)=\frac{5}{6}=0,83 ; \quad M_{S}^{\prime}\left(r_{4}\right)=M_{R}{ }^{\prime}\left(r_{4}\right)=M_{C}{ }^{\prime}\left(r_{4}\right)=\frac{4}{6}=0,66 ; \\
& M_{S}{ }^{\prime}\left(r_{5}\right)=\frac{4}{6}=0,66 ; M_{C}{ }^{\prime}\left(r_{6}\right)=\frac{4}{6}=0,66
\end{aligned}
$$

Taking into account the semantic evaluation criterion, we note that:

- Each rule with the destination port value "ANY" gains importance.

- There are rules involved in several errors. For example, rule $r_{3}$ has an impact on all anomalies categories.

In the next section, we will consider this criterion because it increases the errors severity.

\subsubsection{Multi-anomalies importance evaluation}

Sometimes, a rule is involved in several anomalies. We are talking about "multi-anomalies" categories. In the case of firewall anomalies, there are simple errors category, double errors category, triple errors category and quadruple errors category. For example, as presented in the sub-section 5.2.4, $\mathrm{r}_{3}$ is involved in the shadowing error category, the generalization error category, the correlation error category and the redundancy error category. Also, $r_{2}$ is involved in the shadowing error category and the redundancy error category. These categories are detailed as follows:

- $\quad$ Simple error category: The simple error category is defined as follows:

$$
S M=\left\{r \in(S \vee R \vee G \vee C) \wedge\left[\begin{array}{l}
r_{i} \notin(S \cap R) \wedge r_{i} \notin(S \cap G) \wedge r_{i} \notin(S \cap C) \\
\wedge r_{i} \notin(R \cap G) \wedge r_{i} \notin(R \cap C) \wedge r_{i} \notin(G \cap C)
\end{array}\right]\right\}
$$


International Journal of Network Security \& Its Applications (IJNSA), Vol.5, No.4, July 2013

- $\quad$ Double errors category: The double error category is defined as follows:

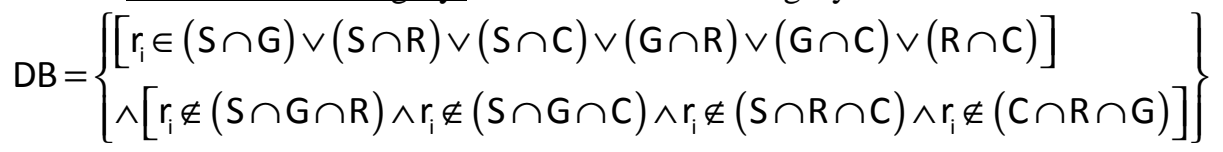

- $\quad$ Triple errors category: The triple error category is defined as follows:

$T R=\left\{r_{i} \in[(S \cap G \cap R) \vee(S \cap G \cap C) \vee(S \cap R \cap C) \vee(G \cap R \cap C)] \wedge r_{i} \notin(S \cap G \cap R \cap C)\right\}$

- $\quad$ Quadruplet errors category: The quadruplet error category is defined as follows:

$Q D=\{S \cap G \cap R \cap C\}(20)$

In order to show the impact of the multi-anomalies categories, the administrator will associate a weight to each category of error. In the case of firewalls, if a rule $r_{i}$ belongs to the SM category, it will associate to it a coefficient $M_{s}^{\prime \prime}\left(r_{i}\right)=M_{G}^{\prime \prime}\left(r_{i}\right)=M_{c}^{\prime \prime}\left(r_{i}\right)=M_{R}^{\prime \prime}\left(r_{i}\right)=0,25$. If a rule $r_{i}$ belongs to the DB category, it will associate to it a coefficient $M_{s}^{\prime \prime}\left(r_{i}\right)=M_{G}^{\prime \prime}\left(r_{i}\right)=M_{R}^{\prime \prime}\left(r_{i}\right)=M_{C}^{\prime \prime}\left(r_{i}\right)=0,5$. If a rule $r_{i}$ belongs to the TR category, it will associate to it a coefficient $M_{S}^{\prime \prime}\left(r_{i}\right)=M_{G}^{\prime \prime}\left(r_{i}\right)=M_{R}^{\prime \prime}\left(r_{i}\right)=M_{C}^{\prime \prime}\left(r_{i}\right)=0,75$ and finally, if a rule $r_{i}$ belongs to the QD category, it will associate to it a coefficient $M_{S}^{\prime \prime}\left(r_{i}\right)=M_{G}^{\prime \prime}\left(r_{i}\right)=M_{C}^{\prime \prime}\left(r_{i}\right)=M_{R}^{\prime \prime}\left(r_{i}\right)=1$.

\subsubsection{Case study}

Based on (1), (2), (3) and (4), rules involving anomalies are $r_{1}, r_{2}, r_{3}, r_{4}, r_{5}$ and $r_{6}$. Applying (17), (18), (19) and (20), in our case study, we have:

$-r_{5} \in S M=\{S\}$, it will have a coefficient $M_{S}^{\prime \prime}\left(r_{5}\right)=0,25$

$-r_{6} \in S M=\{C\}$, it will have a coefficient $M_{C}^{\prime \prime}\left(r_{6}\right)=0,25$

$-r_{1} \in D B=\{S \cap R\}$, it will have a coefficient $M_{S}^{\prime \prime}\left(r_{1}\right)=M_{R}^{\prime \prime}\left(r_{1}\right)=0,5$

$-r_{2} \in D B=\{S \cap R\}$, it will have a coefficient $M_{S}^{\prime \prime}\left(r_{2}\right)=M_{R}^{\prime \prime}\left(r_{2}\right)=0,5$

$-r_{4} \in T R=\{S \cap R \cap C\}$, it will have a coefficient $M_{S}^{\prime \prime}\left(r_{4}\right)=M_{R}^{\prime \prime}\left(r_{4}\right)=M_{C}^{\prime \prime}\left(r_{4}\right)=0,75$

$-r_{3} \in Q D=\{S \cap G \cap R \cap C\}$, it will have a coefficient $M_{S}^{\prime \prime}\left(r_{3}\right)=M_{G}^{\prime \prime}\left(r_{3}\right)=M_{C}^{\prime \prime}\left(r_{3}\right)=M_{R}^{\prime \prime}\left(r_{3}\right)=1$

\section{Classification of THE ANOMAlies importance (STEP C)}

In section 5, we have proposed, for each rule, three metrics $\mathrm{M}, \mathrm{M}^{\prime}$ and $\mathrm{M}^{\prime \prime}$. Gathering these three metrics together give us an interesting measure $\boldsymbol{M M}^{\prime} \boldsymbol{M}^{\prime \prime}$ that we can incorporate either in an audit process or for assessing security component vulnerability.

In this section, we will exploit this measure and classify the anomalies severity importance relatively to the shadowing anomaly importance. The purpose of this classification is to schematize the impact of the shadowing anomaly severity in a rule. Also, this classification will determine the vulnerable services and sources of attack rules. The exploitation of these results will allow the administrator to decide the order of rules correction and review the security of important services. For a significant evaluation, we propose a classification based on acceptable thresholds. In the next section, we will classify only the shadowing anomaly. The same study can 
International Journal of Network Security \& Its Applications (IJNSA), Vol.5, No.4, July 2013

be made for other types of anomalies i.e. the generalization, the redundancy and the correlation anomalies.

\subsection{The shadowing anomaly importance classification}

In this section, we suppose that the administrator has defined acceptable shadowing thresholds $S M_{\mathrm{s}}, S M_{\mathrm{s}}^{\prime}$ and $S M_{\mathrm{s}}^{\prime \prime}$ for respectively $M_{\mathrm{s}}, M_{\mathrm{s}}^{\prime}$ and $M_{\mathrm{s}}^{\prime \prime}$ metrics. According to $M_{\mathrm{s}}, M_{\mathrm{s}}^{\prime}$ and $M_{\mathrm{s}}^{\prime \prime}$ values, we propose the following notations:

- $M_{\mathrm{s}}^{+}$if the value $M_{\mathrm{s}} \leq S M_{\mathrm{s}}$ and $M_{\mathrm{s}}^{-}$if the value $M_{\mathrm{s}}>S M_{\mathrm{s}}$

- $M_{\mathrm{s}}^{\prime}{ }^{+}$if the value $M_{\mathrm{s}}^{\prime} \leq S M_{\mathrm{s}}^{\prime}$ and $M_{\mathrm{s}}^{\prime-}$ if the value $M_{\mathrm{s}}^{\prime}>S M_{\mathrm{s}}^{\prime}$

- $M_{\mathrm{s}}^{\prime \prime}$ if the value $M_{\mathrm{s}}^{\prime \prime} \leq S M_{\mathrm{s}}^{\prime \prime}$ and $M_{\mathrm{s}}^{\prime \prime}$ if the value $M_{\mathrm{s}}^{\prime \prime}>S M_{\mathrm{s}}^{\prime \prime}$

In this way, we can classify the shadowing importance degree compared to the associated $S M_{\mathrm{s}}$, $S M_{\mathrm{S}}^{\prime}$ and $S M_{\mathrm{S}}^{\prime \prime}$ values. The shadowing anomaly importance degree in a rule can belong to one of the eight following classes:

$M_{s}^{+} M_{s}^{\prime+} M_{s}^{\prime \prime}, M_{s}^{-} M_{s}^{\prime+} M_{s}^{\prime+}, M_{s}^{+} M_{s}^{\prime}-M_{s}^{\prime+}, M_{s}^{+} M_{s}^{\prime+} M_{s}^{\prime-}, M_{s}^{-} M_{s}^{\prime+} M_{s}^{\prime \prime}, M_{s}^{-} M_{s}^{\prime}-M_{s}^{\prime+}, M_{s}^{+} M_{s}^{\prime}{ }^{\prime \prime} M_{s}^{-}$, $\mathrm{M}_{\mathrm{S}}^{-} \mathrm{M}_{\mathrm{s}}^{\prime-} \mathrm{M}_{\mathrm{s}}^{\prime-}$

Figure 2, gives a classification of the eight shadowing anomaly importance classes in a rule from the worst one $\left(M_{s}^{-} M_{s}^{\prime} M_{s}^{\prime \prime}\right)$ to the best one $\left(M_{s}^{+} M_{s}{ }^{+} M_{s}^{\prime \prime}\right)$.

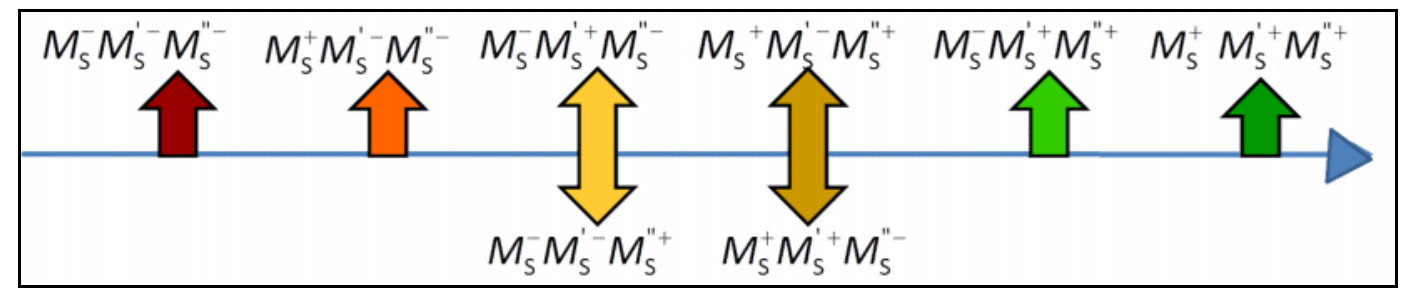

Figure 2. Classification of the shadowing anomaly importance classes

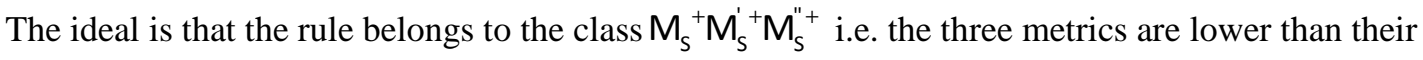
respective thresholds $S M_{\mathrm{S}}, S M_{\mathrm{s}}^{\prime}$ and $S M_{\mathrm{S}}^{\prime \prime}$. A rule belonging to the class $M_{\mathrm{S}}{ }^{-} \mathrm{M}_{\mathrm{S}}^{\prime}{ }^{-} \mathrm{M}_{\mathrm{s}}{ }^{\prime-}$ is a critical rule since all its metrics are higher than their respective thresholds $S M_{\mathrm{S}}, S M_{\mathrm{S}}^{\prime}$ and $S M_{\mathrm{s}}^{\prime \prime}$.

In figure 2, we notice that the measure classes $M_{s}{ }^{+} M_{s}^{\prime}{ }^{-} M_{s}^{\prime+}$ and $M_{s}{ }^{+} M_{s}^{\prime}{ }^{+} M_{s}^{\prime \prime}$ (as far as $M_{s}{ }^{-} M_{s}^{\prime} M_{s}^{\prime-}$ and $M_{s}{ }^{-} M_{s}^{\prime} M_{s}^{\prime \prime}$ ) are classified with the same rank. From our point of view, the semantic measure $M_{\mathrm{s}}^{\prime}$ and the multi-anomalies measure $M_{\mathrm{s}}^{\prime \prime}$ are equivalent considering that they have generally the same importance. However, the administrator can give more importance to one of these two metrics and thus change the classification. 
International Journal of Network Security \& Its Applications (IJNSA), Vol.5, No.4, July 2013

\subsubsection{Case study}

Based on the shadowing evaluation metrics defined above, we suppose that the administrator has fixed the thresholds values as follows: $S M_{\mathrm{s}}=0,2 ; S M_{\mathrm{s}}^{\prime}=0,7$ and $S M_{\mathrm{s}}^{\prime \prime}=0,6$. If we take the shadowing rule $r_{1}$ values: $M_{S}\left(r_{1}\right)=0,333 ; M_{s}^{\prime}\left(r_{1}\right)=0,83$ and $M_{s}^{\prime \prime}\left(r_{1}\right)=0,5$ ( see sections 5.2.2, 5.2.4 and 5.2.6), this latter belongs to the class $M_{s}{ }^{-} M_{s}{ }^{-} M_{s}{ }^{+}$(see figure 3). This class is $5^{\text {th }}$ according to the proposed classification in figure 2 , which implies that the rule $r_{1}$ is critical.

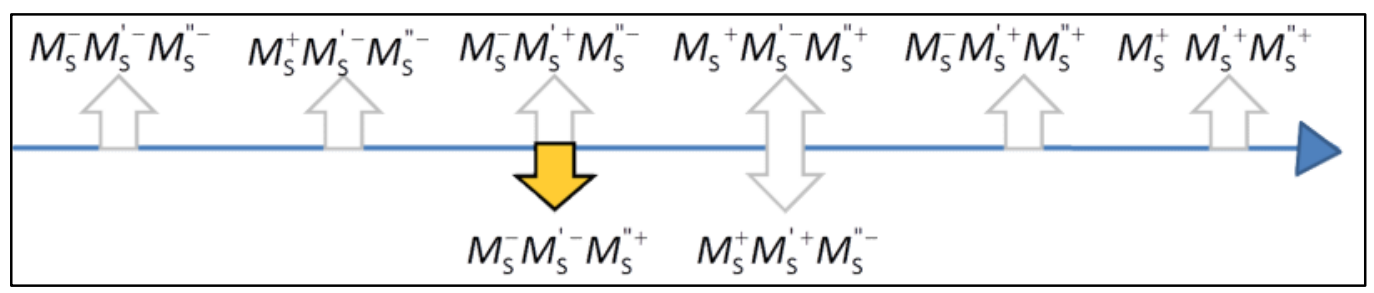

Figure 3 . The rule $r_{1}$ shadowing anomaly importance representation

To further specify the impact of the shadowing rule $r_{1}$, we will illustrate its evaluations values relatively to the acceptable thresholds defined above in a three-dimensional benchmark composed of three axes: the axis $\mathrm{M}_{\mathrm{s}}$, the axis $\mathrm{M}_{\mathrm{s}}$ and the axis $\mathrm{M}_{\mathrm{s}}$. In figure 4, the thresholds values defined above represent the vertexes of the green triangle. The shadowing $r_{1}$ evaluation values $M_{s}\left(r_{1}\right)$, $M_{\mathrm{s}}^{\prime}\left(r_{1}\right)$ and $M_{\mathrm{s}}^{\prime \prime}\left(r_{1}\right)$ represent the vertexes of the red triangle.

Any red triangle included/coinciding in/to the green triangle indicates that its metrics values are acceptables. Exceeding any side of the triangle indicates that the corresponding measure is critical.

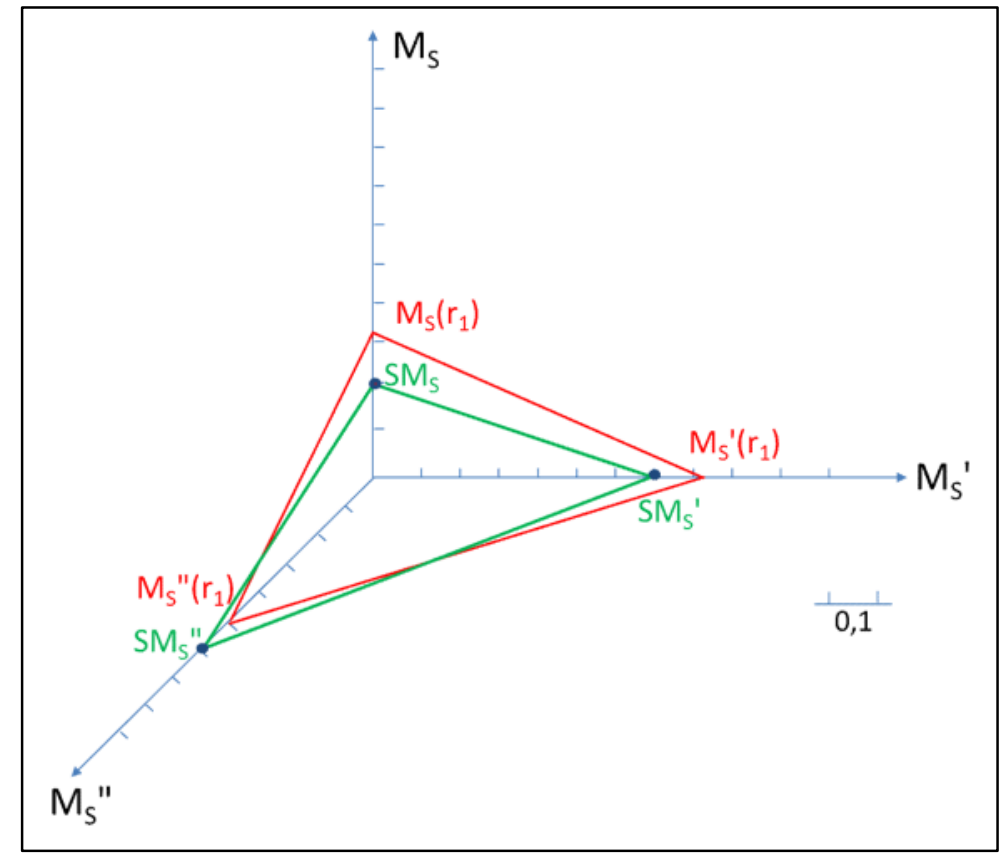

Figure 4. The rule $r_{1}$ shadowing anomaly evaluation metrics representation 
In our case study, the vertexes of the red triangle represent the rule $r_{1}$ evaluation values. We note that these latter exceed those of the green triangle on the side of axes $\mathbf{M}_{\mathrm{s}}$ and $\mathbf{M}_{\mathrm{s}}{ }_{\mathrm{s}}$. Based on results returned in sections 5.2.2 and 5.2.4, this is explained as follows:

-From the side of the axis $\mathrm{M}_{\mathrm{s}}$, the quantitative measure $\mathrm{M}_{\mathrm{s}}\left(\mathrm{r}_{1}\right)$ exceeds the threshold $\mathrm{SM}_{\mathrm{s}}$, this is due to the large number of rules masked by $r_{1}$.

-From the side of the axis $\mathrm{M}_{\mathrm{s}}$, the semantic measure $\mathrm{M}_{\mathrm{s}}{ }_{\mathrm{s}}\left(\mathrm{r}_{1}\right)$ exceeds the threshold $\mathrm{S} \mathrm{M}_{\mathrm{s}}{ }_{\mathrm{s}}$. This is due to the value ANY in the "destination port" attribute, that accepts all incoming flow and therefore, this port can be easily attacked.

\section{CONCLUSION}

In this paper, we evaluated the severity importance of anomalies with 3 manners; a quantitative evaluation, a semantic evaluation and a multi-anomalies evaluation. The quantitative evaluation showed us the number of rules involved in each type of anomaly. However, this first metric lack of semantic i.e. it does not display the impact of the anomaly on the security component base rules. As a complementary metric, we have defined the semantic metric. This metric allows us to determine the degree of vulnerability of each service involved in the anomaly. In addition, we noted that there are rules involved in more than one anomaly. Therefore, we proceeded to a "multi-anomalies" assessment to show the impact of anomalies combination on the rule. In addition, we exploited this information by classifying the impact of these errors on the network security by a set of diagrams.

\section{REFERENCES}

[1] P. Eronen, and J. Zitting, 2001. An Expert System for Analyzing Firewall Rules. Proceedings of 6th Nordic Workshop on Secure IT-Systems.

[2] J. Garcia-Alfaro, F.Cuppens, and N. Cuppens-Boulahia, Analysis of Policy Anomalies on Distributed Network Security Setups. Proceedings of the 11th European Symposium on research in computer security (ESORICS 2006), Hamburg, Germany.

[3] H. Hamed, E. Al-Shaer, and W. Marrero, Modeling and Verification of IPsec and VPN Security Policies. Proc. 13th IEEE Int'l Conf. Network Protocols (ICNP'2005), pp. 259-278.

[4] E. Al-Shaer, H.Hamed, R. Boutaba, M. Hasan, Conflict classification and analysis of distributed firewall policies, IEEE Journal on Selected Areas in Communications (JSAC) 2005, pp. 2069-2084.

[5] E. Al-Shaer, and H. Hamed, Discovery of Policy Anomalies in Distributed Firewalls, Proc. IEEE INFOCOM '2004, pp. 2605-2615.

[6] A.X. Liu, and M. Gouda, Complete Redundancy Detection in Firewalls, Proc. 19th Ann. IFIP Conf. Data and Applications Security (2005), pp. 196-209.

[7] M. Gouda, and A.X. Liu, A Model of Stateful Firewalls and Its Properties, Proc. IEEE Int'l Conf. Dependable Systems and Networks (DSN '2005), pp. 320-327.

[8] SP. Pornavalai, and T. Chomsiri, Analyzing Firewall Policy with Relational Algebra and its Application. Australian Telecommunication Networks and Applications Conference (ATNAC 2004), Australia.

[9] F. Ben Ftima, K. Karoui and H. Ben Ghezala, A multi-agent framework for anomalies detection on distributed firewalls using data mining techniques. Springer Verlag "Data Mining and Multi-agent Integration", 2009. ISBN: 978-1-4419-0523-9, pp267-278.[10]A.X. Liu, Firewall Policy Verification and Troubleshooting, Proc. IEEE Int'l Conf. Comm. (ICC) 2008. 